\title{
Ester-activated vinyl ethers as chain transfer agents in radical photopolymerization of methacrylates
}

Gernot Peer, ${ }^{\dagger,+}$ Anna Eibel, ${ }^{ \pm}$Christian Gorsche, ${ }^{\dagger}$ Yohann Catel, $\|,+$, Georg Gescheidt, ${ }^{ \pm}$Norbert Moszner\|, t; and Robert Liska ${ }^{*}+,+$,

${ }^{\dagger}$ Institute of Applied Synthetic Chemistry, TU Wien, Getreidemarkt 9/163 MC, 1060 Vienna, Austria

$¥$ Christian-Doppler-Laboratory for Photopolymers in Digital and Restorative Dentistry, Getreidemarkt 9, 1060 Vienna, Austria

${ }^{ \pm}$Institute of Physical and Theoretical Chemistry, Graz University of Technology, Stremayrgasse 9, 8010 Graz, Austria

"Ivoclar Vivadent AG, 9494 Schaan, Liechtenstein

*corresponding author: robert.liska@tuwien.ac.at 


\section{Acid stability}

For acid stability tests, vinyl ether EOE was mixed with the acidic monomer (2-((2-(ethoxycarbonyl)allyl)oxy)ethyl)phosphonic acid (ECAP) in an equimolar ratio and the mixture was measured via ${ }^{1} \mathrm{H}-\mathrm{NMR}$ with an equivolumetric mixture of $\mathrm{D}_{2} \mathrm{O}$ and $\mathrm{CDCN}$ as solvent. Additionally the same solvent mixture was used to prepare a $1 \mathrm{~N}$ acetic solution by adding glacial acetic acid. This acetic solvent mixture was used to measure ${ }^{1} \mathrm{H}-\mathrm{NMR}$ spectra of neat EOE. The hydrolysis of EOE in these two different acidic environments was tracked over time via the integral decrease of the double bond signal for EOE. As an internal reference, the signal of the two $-\mathrm{CH}_{3}$ groups was used.

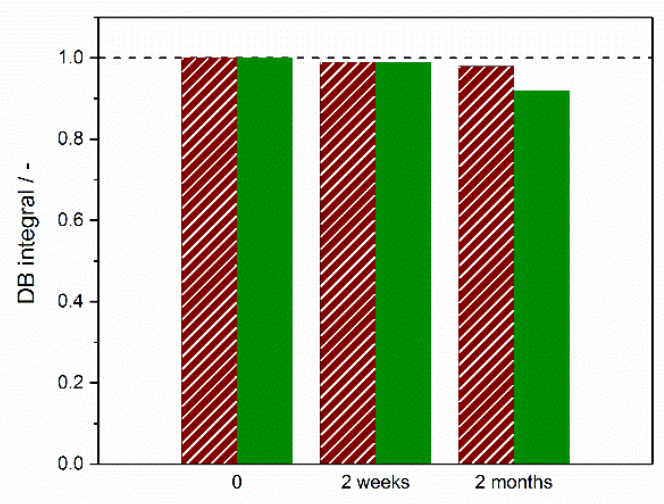

Figure S1. Double bond integral of EOE with ECAP (sparse) and EOE in 1N acetic acid solution (bulk) after 2 weeks and 2 months 


\section{Gel permeation chromatography}

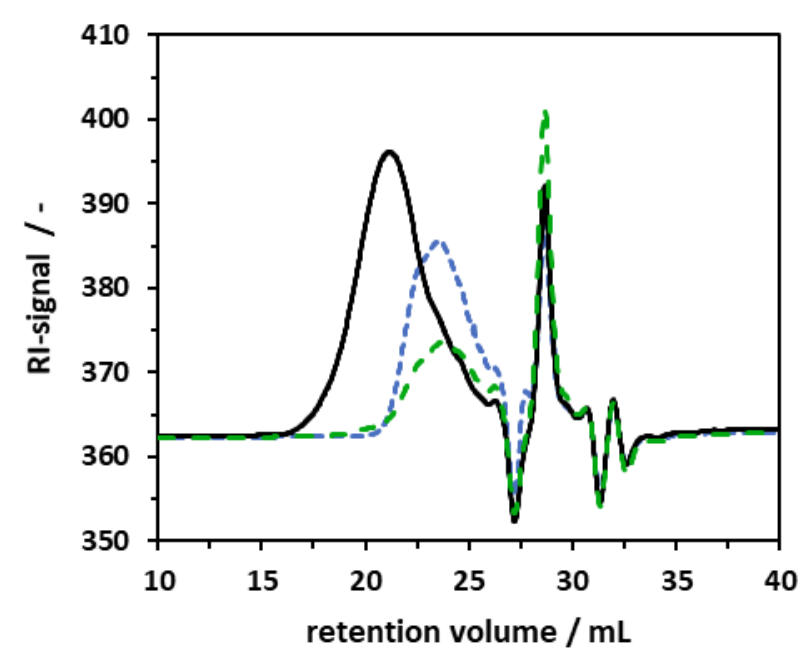

Figure S2. Gel permeation chromatogram of p(BMA) (solid), p(BMA)-(EOE) (dash) and p(BMA)(MMA-D) (dash)

\section{Photoreactor}

For photoreactor studies BMA was used as a monomer. Formulations with 0 and 20 mol\% EOE (BMA_EOE 20) were prepared and $3 \mathrm{~mol} \%$ BMDG as photoinitiator was added. For every measurement $400 \mathrm{mg}$ formulation were placed in a $10 \mathrm{~mL}$ two-necked round bottom flask and were diluted with $2 \mathrm{~mL}$ benzene- $\mathrm{d}_{6}$ as solvent. The solutions were purged with argon for $20 \mathrm{~min}$ before being irradiated with UV-light (Exfo OmniCure S2000 broadband Hg-lamp with a $400-500 \mathrm{~nm}$ filter, $0.5 \mathrm{~cm}^{-2}$ irradiation intensity at the tip of the light guide, $\sim 3 \mathrm{~mW} \mathrm{~cm} \mathrm{co}^{-2}$ on the surface of the formulation). Samples $(0.05 \mathrm{~mL})$ were taken before irradiation and after $50 \mathrm{~s}, 100 \mathrm{~s}$, 200 s, 300 s, 450 s, 600 s, 900 s, 1500 s, 2100 s and $3000 \mathrm{~s}$ of irradiation. The samples were diluted with $0.6 \mathrm{~mL} \mathrm{CDCl}_{3}$ and ${ }^{1} \mathrm{H}-\mathrm{NMR}$ spectra were recorded. The conversion was calculated from the integral ratio between the vinyl signals before irradiation and after the defined periods of time, while the $-\mathrm{CH}_{2}$ - signal from BMA served as reference signal. 


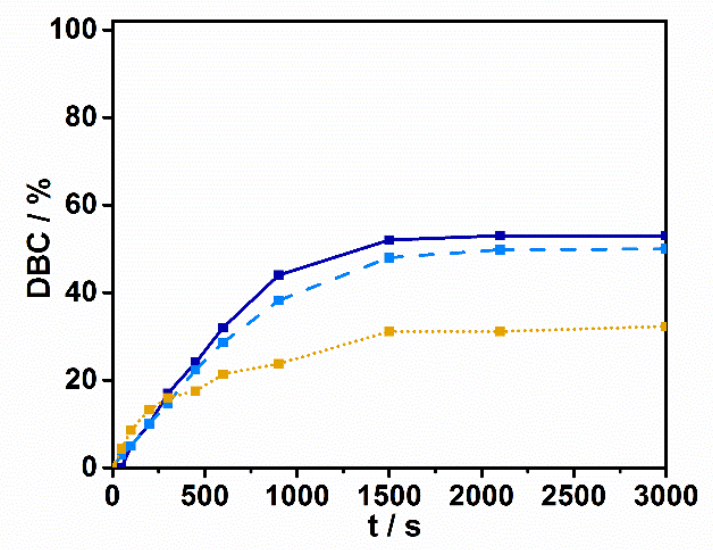

Figure S3. Double bond conversion of BMA (solid) and BMA_EOE 20 (BMA...dash and EOE dot) RT-NIR-Photorheology

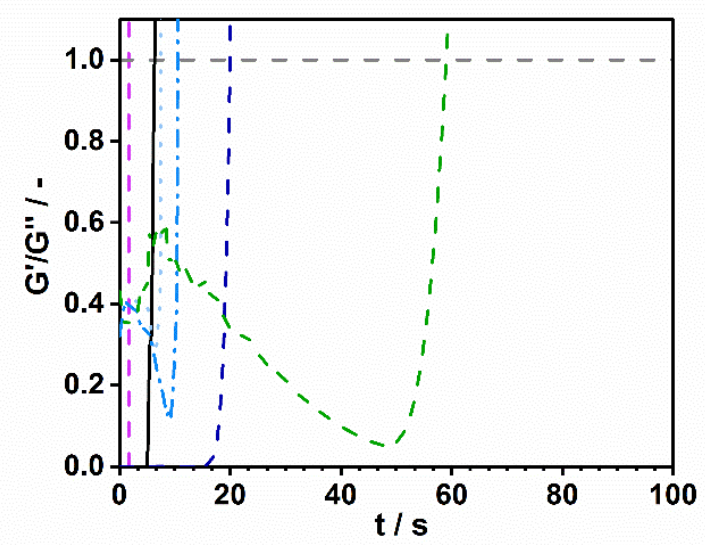

Figure S4. G'/G', over time of 2M (solid), 2M_EOE 5 (dot), 2M_EOE 10 (dash-dot), 2M_EOE 20 (dash) and 2M_MMA-D 20 (dash); (irradiation starts at $5 \mathrm{~s}$, indicated by purple dashed line, $\mathrm{G}^{\overline{ }} / \mathrm{G}^{\prime}$ ' $=1$ marks gelation) 
Table S1. Results from RT-NIR-photorheology with an equimolar mixture of $\mathrm{D}_{3} \mathrm{MA}$ and UDMA and mixtures containing different amounts of AFCT-agents; ( $t_{\text {gel }} \ldots$ time to gel point; $\mathrm{DBC}_{\text {gel }} \ldots$ double bond conversion at the gel point; $\mathrm{DBC}_{\text {final }} \ldots$ final double bond conversion; $\mathrm{t}_{95 \%\left(\mathrm{G}^{\prime}\right)} \ldots$. time to $95 \%$ of final storage

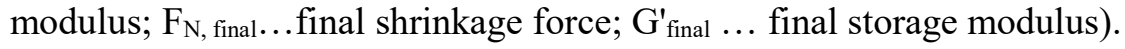

\begin{tabular}{ccccccc}
\hline formulation & $\begin{array}{c}\mathbf{t g e l}_{\text {gel }} \\
/ \mathbf{s}\end{array}$ & $\begin{array}{c}\mathbf{D B C} \text { gel } \\
/ \mathbf{\%}\end{array}$ & $\begin{array}{c}\mathbf{t}_{\mathbf{9}}\left(\mathbf{G}^{\cdot}\right) \\
/ \mathbf{s}\end{array}$ & $\begin{array}{c}\mathbf{D B C}_{\text {Final }} \\
/ \mathbf{\%}\end{array}$ & $\begin{array}{c}\mathbf{F}_{\mathbf{N}} \\
/ \mathbf{N}\end{array}$ & $\begin{array}{c}\mathbf{G}_{\text {Final }} \\
/ \mathbf{M P a}\end{array}$ \\
\hline 2M & $1.3 \pm 0.1$ & $17 \pm 0$ & $41 \pm 3$ & $79.4 \pm 0.5$ & $-32.2 \pm 0.8$ & $0.98 \pm 0.04$ \\
\hline 2M_EOE 5 & $4.3 \pm 0.0$ & $25 \pm 1$ & $70 \pm 1$ & $83.9 \pm 0.6$ & $-31.1 \pm 0.6$ & $1.00 \pm 0.03$ \\
\hline 2M_EOE 10 & $7.0 \pm 0.2$ & $27 \pm 0$ & $96 \pm 10$ & $87.6 \pm 0.2$ & $-29.1 \pm 0.8$ & $1.01 \pm 0.01$ \\
\hline 2M_EOE 20 & $15.3 \pm 0.0$ & $31 \pm 1$ & $202 \pm 5$ & $90.8 \pm 0.3$ & $-25.6 \pm 1.0$ & $0.96 \pm 0.01$ \\
\hline 2M_MMA-D 20 & $54.3 \pm 0.4$ & $17 \pm 0$ & $279 \pm 1$ & $43.3 \pm 0.5$ & $-15.0 \pm 0.2$ & $0.62 \pm 0.01$ \\
\hline
\end{tabular}

For storage stability tests, 2M_EOE 20 was kept in dark for 9 months and afterwards measured via RT-NIR-photorheology with the same parameters as described in the manuscript. 
a)

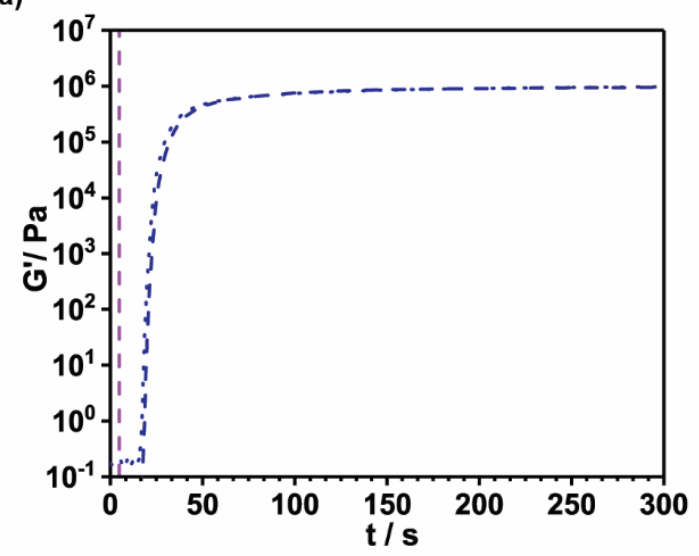

b)

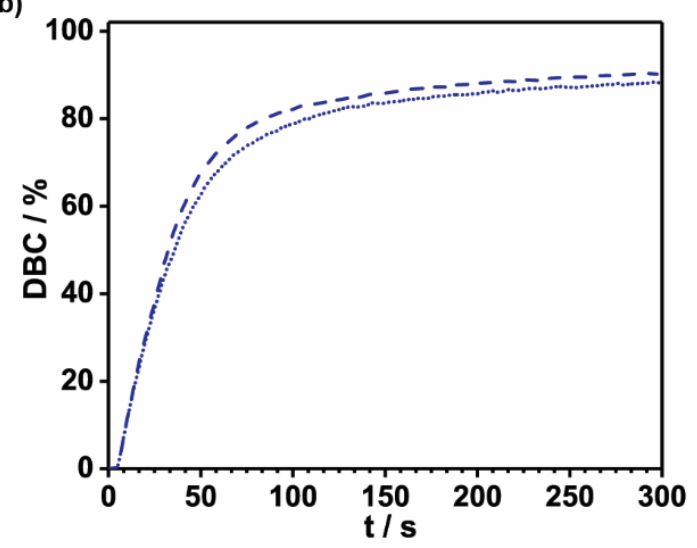

Figure S5. a) Storage modulus G' and b) DBC over time of freshly mixed 2M_EOE 20 (dash) and 2M_EOE 20 after 9 months kept in dark (dot). 


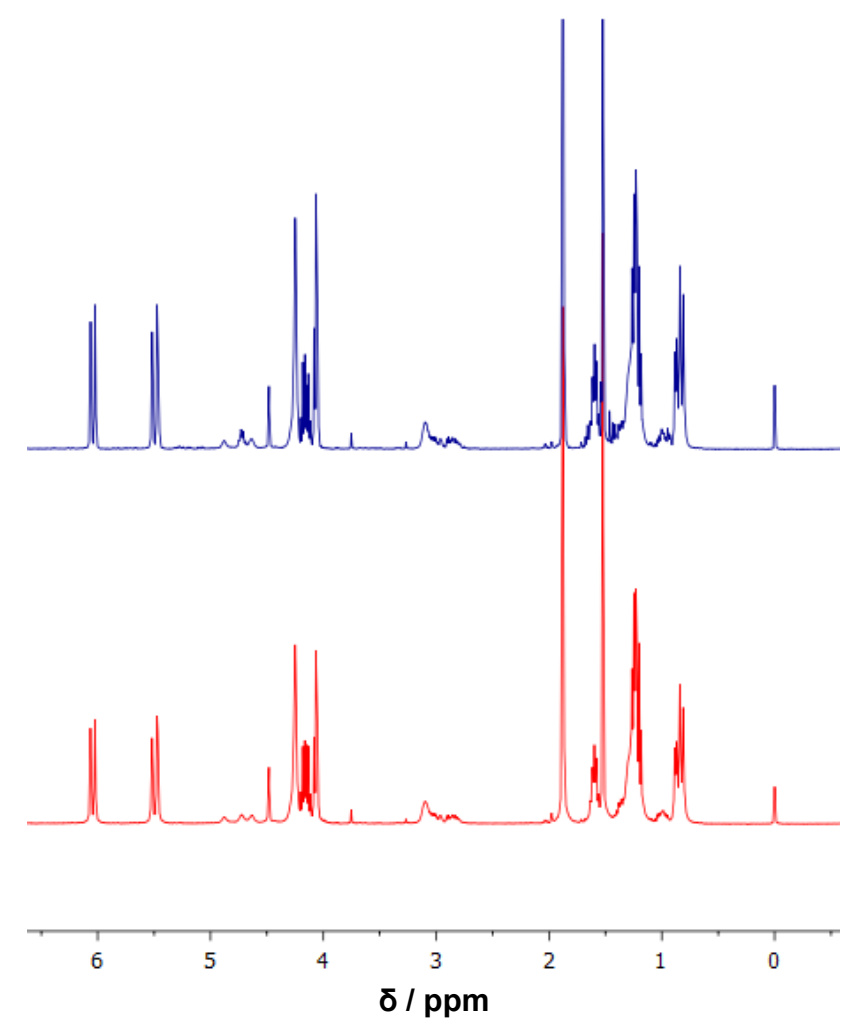

Figure S6. ${ }^{1} \mathrm{H}$ spectra of 2M_EOE 20 before (blue) and after a storage period of 9 months (red) at room temperature

\section{Swellability tests}

Table S2. Swellability and gel fraction poly $(2 \mathrm{M})$ and respective photopolymers with EOE or MMA-D

\begin{tabular}{ccc}
\hline polymer & $\begin{array}{c}\text { swellability } \\
\text { / wt \% }\end{array}$ & $\begin{array}{c}\text { gel fraction } \\
\text { / wt\% }\end{array}$ \\
\hline 2M & $1.82 \pm 0.07$ & $99.05 \pm 0.06$ \\
\hline 2M_EOE 5 & $2.27 \pm 0.15$ & $99.21 \pm 0.08$ \\
\hline 2M_EOE 10 & $3.92 \pm 0.42$ & $99.44 \pm 0.22$ \\
\hline 2M_EOE 20 & $17.70 \pm 1.19$ & $96.39 \pm 0.45$ \\
\hline 2M_MMA-D 20 & $18.85 \pm 0.27$ & $87.33 \pm 0.56$ \\
\hline
\end{tabular}




\section{Dynamic Mechanical Thermal Analysis (DMTA)}

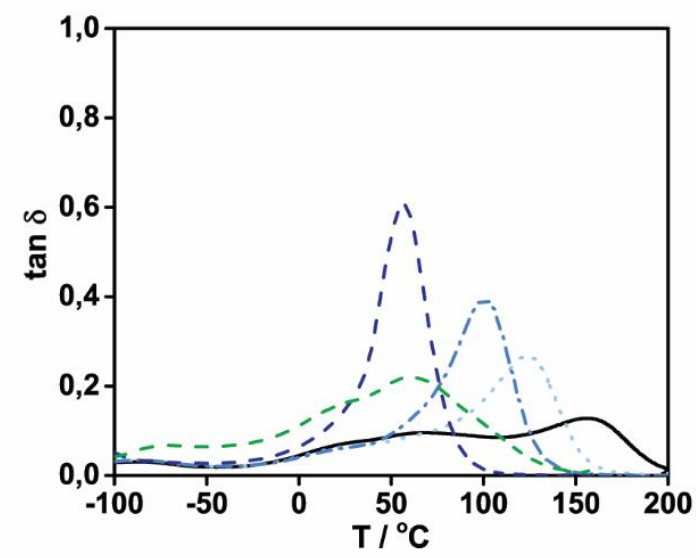

Figure S7. Loss factor (tan $\delta)$ over temperature for poly(2M) (solid), poly(2M_EOE 5) (dot), poly(2M_EOE 10) (dash-dot), poly(2M_EOE 20) (dash) and poly(2M_MMA-D 20) (dash).

Table S3. Results from DMTA for poly $(2 \mathrm{M})$ and respective photopolymers with EOE or MMA-D; $\left(\mathrm{G}^{\prime}{ }_{25} \ldots\right.$... storage modulus at $25^{\circ} \mathrm{C} ; \mathrm{T}_{\mathrm{g}} \ldots$ glass transition temperature derived from the maximum of $\tan \delta$; fwhm...full width at half max of $\tan \delta$ plot; $\mathrm{G}_{\mathrm{r}}{ }_{\mathrm{r}}$... storage modulus at the rubber state).

\begin{tabular}{ccccc}
\hline polymer & $\begin{array}{c}\mathbf{G}_{25} \\
/ \mathbf{M P a}\end{array}$ & $\begin{array}{c}\mathbf{T}_{\mathbf{g}} \\
/{ }^{\circ} \mathbf{C}\end{array}$ & $\begin{array}{c}\mathbf{f w h m}^{\circ} \\
/{ }^{\circ} \mathbf{C}\end{array}$ & $\begin{array}{c}\mathbf{G}_{\mathbf{r}} \\
/ \mathbf{M P a}\end{array}$ \\
\hline 2M & 1550 & 156 & - & 161 \\
\hline 2M_EOE 5 & 1470 & 124 & 52 & 65 \\
\hline 2M_EOE 10 & 1460 & 100 & 42 & 36 \\
\hline 2M_EOE 20 & 840 & 57 & 31 & 14 \\
\hline 2M_MMA-D 20 & 379 & 60 & 101 & 55 \\
\hline
\end{tabular}




\section{Tensile Tests}

Table S4. Results from tensile tests for poly(2M) and respective photopolymers with EOE or MMA-D; $\left(\sigma_{\max } \ldots\right.$ maximum stress).

\begin{tabular}{ccc}
\hline polymer & $\begin{array}{c}\boldsymbol{\sigma}_{\max } \\
/ \mathbf{M P a}\end{array}$ & $\begin{array}{c}\text { strain at break } \\
\text { / \% }\end{array}$ \\
\hline 2M & $66.4 \pm 5.4$ & $7.7 \pm 0.8$ \\
\hline 2M_EOE 5 & $68.3 \pm 0.5$ & $10.2 \pm 1.2$ \\
\hline 2M_EOE 10 & $63.4 \pm 2.5$ & $13.5 \pm 1.9$ \\
\hline 2M_EOE 20 & $31.0 \pm 1.0$ & $26.9 \pm 2.8$ \\
\hline 2M_MMA-D 20 & $21.8 \pm 2.7$ & $11.5 \pm 2.9$ \\
\hline
\end{tabular}

\section{Dynstat impact tests}

Table S5. Results from Dynstat impact tests for poly(2M) and respective photopolymers with EOE or MMA-D.

\begin{tabular}{cc}
\hline polymer & $\begin{array}{c}\text { impact resistance } \\
/ \mathbf{~ k J ~ ~}^{-2}\end{array}$ \\
\hline 2M & $5.9 \pm 2.3$ \\
\hline 2M_EOE 5 & $9.3 \pm 1.6$ \\
\hline 2M_EOE 10 & $11.5 \pm 1$ \\
\hline 2M_EOE 20 & $12.9 \pm 2.3$ \\
\hline 2M_MMA-D 20 & $6.1 \pm 2.1$ \\
\hline
\end{tabular}

